\title{
THE COHOMOLOGY OF AUGMENTED ALGEBRAS AND GENERALIZED MASSEY PRODUCTS FOR DGA-ALGEBRAS
}

\author{
BY \\ J. PETER MAY(1)
}

We will here describe a new procedure for the calculation of the cohomology $H^{*}(A)=\operatorname{Ext}_{A}(K, K)$ of a graded augmented connected $\left(A_{0}=K\right)$ algebra $A$ of finite type over a field $K$. Our procedure, which is developed in the first half of the paper, involves the application of a spectral sequence defined for any DGAalgebra $U$ to the special case $U=C(A)$, the cobar construction of $A$. In the second half of the paper we define Massey products in $U$ and relate them to the differentials in the cited spectral sequence.

To motivate the construction, we recall that any basis for $H_{1}(A)=\operatorname{Tor}_{1,{ }^{*}}(K, K)$ is in one-to-one correspondence with a minimal set of generators of $A$ and any basis for $H_{2}(A)$ is in one-to-one correspondence with a minimal defining set of relations for $A$ (see Wall [11, pp. 440-442]). Now the same result holds for bigraded augmented connected algebras, and in particular for $H^{*}(A)$. Thus knowledge of $H_{1}\left(H^{*}(A)\right)$ and $H_{2}\left(H^{*}(A)\right)$ would essentially determine $H^{*}(A)$. We will prove the following theorem.

THEOREM 1. There exists a spectral sequence $\left\{E^{r} C(A)\right\}$ of differential coalgebras having the properties:

(i) $E_{p, q}^{2} C(A)=H_{p, q}\left(H^{*}(A)\right.$ ) as a graded $K$-module (where $H^{*}(A)$ is graded with lower indices so that $\left.q \leqq 0: H^{*}(A)_{q}=H^{-q}(A)\right)$;

(ii) $E^{2} C(A)=H_{*}\left(H^{*}(A)\right)$ as a coalgebra;

(iii) The differentials satisfy $\partial_{r}: E_{p, q}^{r} C(A) \rightarrow E_{p-r, q+r-1}^{r} C(A)$;

(iv) $E_{p, q}^{\infty} C(A)=0$ if $q \neq-p$;

(v) $E_{p,-p}^{\infty} C(A)=\left(E_{-p, *}^{0} A\right)^{*}$, where $E^{0} A$ denotes the associated graded algebra of $A$ with respect to the augmentation ideal filtration $F_{-p} A=(I A)^{p}$ if $p>0, F_{-p} A=A$ if $p \leqq 0$;

(vi) $E^{\infty} C(A)=\left(E^{0} A\right)^{*}$ as a coalgebra.

By (i), $E_{p, q}^{2} C(A)=0$ if $p<0$ or if $p+q>0$. In principle, knowing $H^{1}(A)$ and the relations in $H^{2}(A)$, we can calculate $E_{p,-p}^{2} C(A)$ for all $p$. Calculation of

Received by the editors September 7,1965.

(1) During the preparation of this paper, the author was partially supported by National Science Foundation grant number NSF-GP-4037. 
the differentials defined on the $E_{p,-p}^{r} C(A)$ determines $H_{1}\left(H^{2}(A)\right.$ ) (the indecomposable elements of $H^{2}(A)$ ), and $H_{2}\left(H^{3}(A)\right.$ ) (the relations in $H^{3}(A)$ ). This knowledge allows the computation of $E_{p,-p-1}^{2} C(A)$, and so forth. Thus an inductive determination of $H^{*}(A)$ is possible. In practice, this procedure is a good deal simpler than direct computation using the cobar construction. In the case of Hopf algebras, the procedure seems slightly less workable than that described by the author in [6].

The differentials $\partial_{r}: E_{r+1, q}^{r} C(A) \rightarrow E_{1, q+r-1}^{r} C(A)$ define cohomology operations with values in the indecomposable elements of $H^{*}(A)$. Even in cases where $H^{*}(A)$ is computed primarily by other methods, these operations should be of value as an auxiliary to the calculation and as a means of interpreting the results. This is a particularly so, as these operations are not too unmanageable algebraically and, as will be shown in Theorem 6, are closely related to Massey products [5]. The method of computation of the differentials (Lemma 4) will make clear how to construct many other explicit operations satisfying the analog of Theorem 6 . This fact may be useful in the study of the Adams spectral sequence [1], since corresponding stable homotopy operations can be defined. For example, the homotopy analog of the Massey triple product is the Toda bracket [10], and the convergence of Massey products to Toda brackets in the Adams spectral sequence has been proven by M. Moss (Cambridge thesis). The homotopy analog of the quadruple product has been studied by Mimura [7] and Oguchi [8], and analogs of the higher operations have been defined by Gershenson [3].

The proof of Theorem 1 is quite simple, requiring only the algebraic machinery developed in MacLane [4, pp. 301-315, 340-342]. To clarify the grading, we outline the construction. Consider $C(A)$ as the dual of the reduced bar construction $\bar{B}(A)=T(s I A)$, the tensor algebra on a copy of $I A$ in which all elements have a homological degree of one. Bigrade $C(A)$ by $C_{q}^{t}(A)=\left[\bar{B}_{-q, t}(A)\right]^{*}$, where $t$ is the degree induced by the grading of $A$ and $-q$ is the homological degree in $\bar{B}(A)$ ) (so that $q \leqq 0) . C(A)=T\left(s^{-1}(I A)^{*}\right)$ as an algebra, and is a DGA-algebra with differential $\delta: C_{q}^{t}(A) \rightarrow C_{q-1}{ }^{t}(A)$ defined on generators $\left[x^{*}\right], x^{*}$ an element of a basis for $(I A)^{*}$ dual to a given basis for $I A$, by

$$
\delta\left[x^{*}\right]=\sum_{i}(-1)^{\operatorname{deg}\left[y_{i}^{*}\right]}\left[y_{i}^{*} \mid z_{i}^{*}\right],
$$

where $\phi^{*}\left(x^{*}\right)=\Sigma_{i} y_{i}^{*} \otimes z_{i}^{*}, \phi: A \otimes A \rightarrow A$ being the product in $A$. Now form $\bar{B}(C(A))=T(\operatorname{sIC}(A)) . \bar{B}(C(A))$ is trigraded, with degrees $t$ and $q$ induced from those of $C(A)$ and with a new homological degree $p$. Regrade by the sum of the degrees $p$ and $q, \bar{B}_{n}^{t}(C(A))=\oplus_{p+q=n} \bar{B}_{p, q}^{t}(C(A))$. Except in signs, we may now safely ignore the degree t. $\bar{B}(C(A))$, indeed $\bar{B}(U)$ where $U$ is any DGA-algebra (graded on the nonnegative or the nonpositive integers) is a differential coalgebra with differential $\partial=\partial^{\prime}+\partial^{\prime \prime}$ and coproduct $\psi$, where 


$$
\begin{aligned}
& \partial^{\prime}\left[a_{1}|\cdots| a_{p}\right]=\sum_{i=1}^{p-1}(-1)^{\lambda(i)}\left[a_{1}|\cdots| a_{i} a_{i+1}|\cdots| a_{p}\right], \\
& \partial^{\prime \prime}\left[a_{1}|\cdots| a_{p}\right]=-\sum_{i=1}^{p}(-1)^{\lambda(i-1)}\left[a_{1}|\cdots| \delta a_{i}|\cdots| a_{p}\right],
\end{aligned}
$$

and

$$
\psi\left[a_{1}|\cdots| a_{p}\right]=\sum_{i=0}^{p}\left[a_{1}|\cdots| a_{i}\right] \otimes\left[a_{i+1}|\cdots| a_{p}\right],
$$

$$
\text { for } a_{i} \in U \text { and } \lambda(i)=\operatorname{deg}\left[a_{1}|\cdots| a_{i}\right] \text {. }
$$

We denote by $D H^{*}(U)$ the resulting homology of $\bar{B}(U)$. Filter $\bar{B}(U)$ by $F_{p} \bar{B}_{n}(U)=\bigotimes_{i \leqq p} \bar{B}_{i, n-i}(U)$. The differential $\partial_{0}$ in the resulting spectral sequence $\left\{E^{r} U\right\}$ is induced by $\partial^{\prime \prime}$, and, using the Künneth theorem, it is easily verified that $E^{1} U=\bar{B}(H(U))$ as a differential coalgebra, where $H(U)$ denotes the homology of $U$ regarded as a complex. Since $H(C(A))=H^{*}(A)$, parts (i), (ii), and (iii) of Theorem 1 follow immediately.

$A$ is of finite type, so our spectral sequence converges to $E^{\infty} C(A)=E^{0} D H_{*}(C(A))$. To compute this, we study the spectral sequence $\left\{\tilde{E}^{r} C(A)\right\}$ obtained from the filtration of $\bar{B}(C(A))$ defined by $\tilde{F}_{q} \bar{B}_{n}(C(A))=\bigoplus_{j \leqq q} \bar{B}_{n-j, j}(C(A))$. Here $\tilde{\partial}_{0}$ is induced by $\partial^{\prime}$, hence $\widetilde{E}^{1} C(A)=H_{*}(C(A))$, the homology of $C(A)$ regarded as an algebra. However, the homology of a tensor algebra is easily computed. We find $\tilde{E}_{p, q}^{1} C(A)=0$ except for the cases $\widetilde{E}_{0,0}^{1} C(A)=K$ and $\tilde{E}_{1,-1}^{1} C(A)=(I A)^{*}$ (with typical elements $\left.\left[\left[x^{*}\right]\right], x^{*} \in(I A)^{*}\right)$. Necessarily $\tilde{E}^{1} C(A)=\tilde{E}^{\infty} C(A)$, and therefore $D H^{*}(C(A)) \cong A^{*}$ as a $K$-module. We claim that this is true comultiplicatively. To see this, we prove the dual statement.

Lemma 2. $D H^{n}(C(A))=0$ if $n \neq 0$ and $D H^{0}(C(A)) \cong A$ as an algebra.

Proof. We already know that $D H^{n}(C(A))=0$ if $n \neq 0$ and that $D H^{0}(C(A)) \cong A$ as a $K$-module. Consider $C(C(A))$; multiplicatively $C(C(A))=T\left(s^{-1} I \bar{B}(A)\right)$, suitably graded. Observe that if $x_{i} \in I A$, then:

$$
\begin{aligned}
& \partial^{*}\left[\left[x_{1} \cdots x_{j} \mid x_{j+1}\right]\left|\left[x_{j+2}\right]\right| \cdots \mid\left[x_{n}\right]\right] \\
&=(-1)^{\operatorname{deg} x_{1} \ldots x_{j}}\left[\left[x_{1} \cdots x_{j}\right]\left|\left[x_{j+1}\right]\right|\left[x_{j+2}\right]|\cdots|\left[x_{n}\right]\right] \\
&+(-1)^{\operatorname{deg} x_{1} \ldots x_{j}}\left[\left[x_{1} \cdots x_{j+1}\right]\left|\left[x_{j+2}\right]\right| \cdots \mid\left[x_{n}\right]\right] .
\end{aligned}
$$

It follows easily that every element of $C(C(A)$ in degree $p+q=0$ is congruent to an element of the form $\left[\left[x_{1} \cdots x_{n}\right]\right]$ and in particular that $\left[\left[x_{1}\right]|\cdots|\left[x_{n}\right]\right]$ $\equiv(-1)^{n+1}\left[\left[x_{1} \cdots x_{n}\right]\right]$. We conclude that $f(x)=-[[x]], x \in I A$, defines an epimorphism of algebras $A \rightarrow D H^{0}(C(A))$. But $f$ must then be an isomorphism.

A comparison of the filtration of $\bar{B}(C(A))$ defining $\left\{E^{r} C(A)\right\}$ and of the filtration of $A$ defined in (v) of Theorem 1 now shows that $E_{\infty} C(A)$ of the dual spectral 
sequence is isomorphic to $E^{0} A$ as an algebra. Thus the proof of Theorem 1 is complete.

REMARKS 3. By [4, pp. 312-313], if $U$ is a commutative DGA-algebra, then $\bar{B}(U)$ is a commutative DGA-algebra under the shuffle product.

$$
\left[a_{1}|\cdots| a_{m}\right]^{*}\left[a_{m+1}|\cdots| a_{m+n}\right]=\sum_{\pi}(-1)^{\sigma(\pi)}\left[a_{\pi(1)}|\cdots| a_{\pi(m+n)}\right]
$$

where the sum is taken over all $(m, n)$-shuffles $\pi$ and $\sigma(\pi)=\sum \operatorname{deg}\left[a_{i}\right] \operatorname{deg}\left[a_{m+j}\right]$ summed over all pairs $(i, m+j)$ such that $\pi(i)>\pi(m+j)$.

In fact, $\bar{B}(U)$ is then a differential Hopf algebra (in the sense that both the product and coproduct commute with the differential). Also, by $[4$, p. 310] or the comparison theorem for spectral sequences, if $f: U \rightarrow V$ is a homology isomorphism of DGA-algebras, then $\left\{E^{r} f\right\}$ is an isomorphism of spectral sequences. Thus if there exists a homology isomorphism of DGA-algebras $C(A) \rightarrow U$, where $U$ is commutative, then $\left\{E^{r} C(A)\right\} \cong\left\{E^{r} U\right\}$ is a spectral sequence of differential Hopf algebras. By [6], this is true if $A$ is the universal enveloping algebra of a Lie algebra or of a characteristic two restricted Lie algebra. If $A$ is any Hopf algebra, then $H^{*}(A)$ is commutative and therefore $E^{1} C(A)=\bar{B}\left(H^{*}(A)\right)$ is a differential Hopf algebra, but $\left\{E^{r} A\right\}$ need not, I believe, be a spectral sequence of differential algebras.

We now consider the differentials in the spectral sequence $\left\{E^{r} U\right\}$, and show that those differentials landing in $E_{1, *}^{r} U$ might well be called generalized Massey products. For generality, we assume from now on that $U$ is a DGA-algebra (with differential $\delta$ ) over a commutative ring $\Lambda .\left\{E^{r} U\right\}$ is defined exactly as above, but of course $E^{1} U \neq \bar{B}(H(U))$ in general. If $\alpha_{i} \in H(U)$, the symbol $\left[\alpha_{1}|\cdots| \alpha_{p}\right]$ will denote the image in $E^{1} U$ of $\alpha_{1} \otimes \cdots \otimes \alpha_{p}$ under the obvious (Künneth) map $\bigotimes^{p} H(U) \rightarrow H\left(\otimes^{p} U\right)=E_{p, *}^{1} U$.

We will need the following lemma, which describes the method by which the differentials in $\left\{E^{r} U\right\}$ are to be computed.

LEMMA 4. Let $0 \neq \theta \in E_{p, q}^{r} U, r \geqq 1$, and suppose that $\phi_{1} \in \bar{B}_{p, q}(U)$ is a representative chain for $\theta$, in the sense that $\partial^{\prime \prime} \phi_{1}=0$ and $\left\{\phi_{1}\right\} \in E_{p, q}^{1} U$ survives to 0 . Then there exist $\phi_{i+1} \in \bar{B}_{p-i, q+i}(U), 1 \leqq i \leqq r-1$, such that $\partial^{\prime \prime} \phi_{i+1}+\partial^{\prime} \phi_{i}=0$, and $\partial_{r} \theta$ is that element (possibly zero) to which $\left\{\partial^{\prime} \phi_{r}\right\} \in E_{p-r q+r-1}^{1} U$ survives.

Proof. Note first that it suffices to prove the existence of the $\phi_{i}$, for then $\partial\left(\sum_{i=1}^{r} \phi_{i}\right)=\partial^{\prime} \phi_{r}$ and by definition $\left\{\partial^{\prime} \phi_{r}\right\} \in E^{1} U$ survives to $\partial_{r} \theta$. If $r=1$, the result is trivial. Assume the result for $s<r, r>1$. Since $\left\{\phi_{1}\right\}$ survives to $\eta$, say, in $E^{r-1} U$, there exist $\phi_{i+1} \in \bar{B}_{p-i, q+i}(U), 1 \leqq i \leqq r-2$, such that $\partial^{\prime \prime} \phi_{i+1}+\partial^{\prime} \phi_{i}=0$ and $\left\{\partial^{\prime} \phi_{r-1}\right\} \in E^{1} U$ survives to $\partial_{r-1} \eta=0$. If $\left\{\partial^{\prime} \phi_{r-1}\right\}=0$ in $E^{1} U$, then clearly there exists $\phi_{r}$ such that $\partial^{\prime \prime} \phi_{r}+\partial^{\prime} \phi_{r-1}=0$. Suppose, on the other hand, that $\left\{\partial^{\prime} \phi_{r-1}\right\} \neq 0$ in $E^{1} U$. Then $\left\{\partial^{\prime} \phi_{r-1}\right\}$ must have been killed by some $\partial_{s} \lambda$, $1 \leqq s<r-1$. Again by induction, there exist $\psi_{i}, 1 \leqq i \leqq s$, such that $\partial^{\prime \prime} \psi_{1}=0$, 
$\partial^{\prime \prime} \psi_{i+1}+\partial^{\prime} \psi_{i}=0$, and $\left\{\partial^{\prime} \psi_{s}\right\}=\left\{\partial^{\prime} \phi_{r-1}\right\}$ in $E^{s} U$. Let $\chi_{i}=\phi_{i}, 1 \leqq i<r-s$, and $\chi_{i}=\phi_{i}-\psi_{i+1+s-r}, r-s \leqq i \leqq r-1$. Now either there exists $\chi_{r}$ such that $\partial^{\prime \prime} \chi_{r}=\partial^{\prime}\left(\psi_{s}-\phi_{r-1}\right)=-\partial^{\prime} \chi_{r-1}$, in which case the $\chi_{i}$ satisfy the conclusion, or else $\left\{\partial^{\prime} \chi_{r-1}\right\}$ must have been killed by some $\partial_{t} \lambda^{\prime}, 1 \leqq t<s$, in which case we iterate the argument until the result is obtained.

We remark that the lemma (suitably rephrased) is actually valid for the first spectral sequence of any bicomplex [3, p. 341].

We now recall the definition of Massey $n$-tuple products. In Definitions 5 and 10 and Example 12 below, if $a \in U$ the symbol $\bar{a}$ will denote $(-1)^{1+\operatorname{deg} a} a$.

Definit ION 5. Let $\alpha_{1}, \cdots, \alpha_{n} \in H(U)$. Suppose that $a_{i, i} \in U$ is a representative cycle for $\alpha_{i}$ and suppose there exist chains $a_{i, j}, 1 \leqq i<j \leqq n, j-i<n-1$, such that

$$
\delta a_{i, j}=\sum_{k=1}^{j-1} \bar{a}_{i, k} a_{k+1, j} .
$$

Observe that $\delta a_{1, n}=0$, where

$$
a_{1, n}=\sum_{k=1}^{n-1} \bar{a}_{1, k} a_{k+1, n} .
$$

Under these hypotheses, the Massey $n$-tuple product $\left\langle\alpha_{1}, \cdots, \alpha_{n}\right\rangle$ is defined and is the set of all homology classes $\left\{a_{1, n}\right\}$ so obtainable. $\left(\left\langle\alpha_{1}, \cdots, \alpha_{n}\right\rangle\right.$ is said to be strictly defined if $\left\langle\alpha_{i}, \cdots, \alpha_{j}\right\rangle=\{0\}, j-i<n-1$.)

THEOREM 6. Let $\alpha_{1}, \cdots, \alpha_{r+1} \in H(U)$. Suppose that $\left\langle\alpha_{1}, \cdots, \alpha_{r+1}\right\rangle$ is defined and contains $\beta$ and that $\left[\alpha_{1}|\cdots| \alpha_{r+1}\right] \in E^{1} U$ survives to a nonzero element $\theta \in E^{r} U$. Then $\partial_{r} \theta$ is that element (possibly zero) of $E^{1} U$ to which $[\beta] \in E^{1} U$ survives.

Proof. Using Definition 5, we can easily define $\phi_{i}, 1 \leqq i \leqq r$, such that $\partial^{\prime \prime} \phi_{1}=0$ and $\left\{\phi_{1}\right\}=\left[\alpha_{1}|\cdots| \alpha_{r+1}\right] \in E^{1} U, \partial^{\prime \prime} \phi_{i+1}+\partial^{\prime} \phi_{i}=0$, and $\left\{\partial^{\prime} \phi_{r}\right\}=[\beta] \in E^{1} U$. The result now follows from Lemma 4 .

Definition 7. Suppose that $\left\langle\alpha_{1}, \cdots, \alpha_{r+1}\right\rangle$ is defined and let

$$
\phi=\left[a_{1}|\cdots| a_{r+1}\right] \in \bar{B}(U),
$$

where $a_{i} \in U$ represents $\alpha_{i}$. We say that $\left\langle\alpha_{1}, \cdots, \alpha_{r+1}\right\rangle$ is a good Massey product if there exist no elements $\chi$ and $\psi$ in $\bar{B}(U)$ such that $\partial^{\prime \prime} \chi=\phi-\partial^{\prime} \psi$.

Using Lemma 4 (inductively), it is easily verified that if $\left\langle\alpha_{1}, \cdots, \alpha_{n}\right\rangle$ is a good Massey product, then $\left[\alpha_{1}|\cdots| \alpha_{n}\right]$ is not killed by any $\partial_{s} \lambda, 1 \leqq s$. Therefore we have

Corollary 8. Let $\left\langle\alpha_{1}, \cdots, \alpha_{r+1}\right\rangle$ be a good Massey product and let $\beta \ni\left\langle\alpha_{1}, \cdots, \alpha_{r+1}\right\rangle$. Then $\left[\alpha_{1}|\cdots| \alpha_{r+1}\right]$ survives to $\theta \in E^{r} U, \theta \neq 0$, and either $[\beta] \in E^{1} U$ survives to $\partial_{r} \theta \neq 0$ or else $\theta$ survives to a nonzero element of $E^{\infty} U$. 
Reverting for a moment to the hypotheses of Theorem 1, we have

Corollary 9. Let $\alpha_{i} \in H^{q_{i}}(A), 1 \leqq i \leqq r+1, q_{i} \geqq 1$, and suppose at least one $\left.q_{i}\right\rangle$. Then if $\left\langle\alpha_{1}, \cdots, \alpha_{r+1}\right\rangle$ is a good Massey product, every element $\beta \in\left\langle\alpha_{1}, \cdots, \alpha_{r+1}\right\rangle$ is indecomposable (has nonzero image in $Q H^{*}(A)$ $\left.=I H^{*}(A) /\left(I H^{*}(A)\right)^{2}\right)$ and $\left\langle\alpha_{1}, \cdots, \alpha_{r+1}\right\rangle$ is well defined as an elemnt of $Q H^{*}(A)$. Further, if $\left\langle\alpha_{1}^{\prime}, \cdots, \alpha_{s+1}^{\prime}\right\rangle$ is another good Massey product, $r<s$, then $\beta \notin\left\langle\alpha_{1}^{\prime}, \cdots, \alpha_{s+1}^{\prime}\right\rangle$.

Proof. As follows directly from the definition, any Massey product is well defined as an element of $Q H^{*}(A)$. Now since $\left[\alpha_{1}|\cdots| \alpha_{r+1}\right]$ survives to $\theta \in E_{p, q}^{r} C(A)$, where $\theta \neq 0$ and $q<p$, we must have $\partial_{r} \theta \neq 0$. Therefore [ $\left.\beta\right]$ must survive to a nonzero element of $E_{1,}^{2}, C(A)=H_{1}\left(H^{*}(A)\right)$, hence $\beta$ is indecomposable. The last statement follows, since if $\beta$ were in $\left\langle\alpha_{1}^{\prime}, \cdots, \alpha_{s+1}^{\prime}\right\rangle$, then $\left[\alpha_{1}^{\prime}|\cdots| \alpha_{s+1}^{\prime}\right]$ would survive to a nonzero element of $E^{\infty} C(A)$.

We cite another explicit operation, due (in homotopy theory) to Barratt [2], which satisfies an analog of Theorem 6 .

Definition 10. Let $\alpha, \beta_{1}, \beta_{2}, \gamma_{1}, \gamma_{2} \in H(U)$. Suppose that $a, b_{1}, b_{2}, c_{1}, c_{2} \in U$ are representative cycles and suppose there exist chains $d_{1}, d_{2}$, and $e$ such that

$$
\delta d_{i}=\bar{a} b_{1}, \quad \delta d_{2}=\bar{a} b_{2}, \quad \text { and } \delta e=\tilde{b}_{1} c_{1}+\bar{b}_{2} c_{2} .
$$

Observe that $\delta f=0$, where

$$
f=\bar{d}_{1} c_{1}+\bar{d}_{2} c_{2}+\bar{a} e .
$$

Under these hypotheses, $\left\langle\alpha,{ }_{\beta_{2}, \gamma_{2}}^{\beta_{1}, \gamma_{1}}\right\rangle$ is defined and is the set of all homology classes $\{f\}$ so obtainable.

Proposition 11. Let $\alpha, \beta_{1}, \beta_{2}, \gamma_{1}, \gamma_{2} \in H(U)$. Suppose that $\left\langle\alpha, \alpha_{\beta_{2}, \gamma_{2}}^{\beta_{1} \gamma_{1}}\right\rangle$ is defined and contains $\phi$ and that $\left[\alpha\left|\beta_{1}\right| \gamma_{1}\right]+\left[\alpha\left|\beta_{2}\right| \gamma_{2}\right] \in E^{1} U$ survives to a nonzero element $\theta \in E^{2} U$. Then $\partial_{2} \theta$ is that element (possibly zero) of $E^{2} U$ to which $[\phi] \in E^{1} U$ survives.

We can, of course, complete the analogy by defining "good" operations $\left\langle\alpha, \alpha_{\beta_{2}, \gamma_{2}}^{\beta_{1}}\right\rangle$ and proving analogs of Corollaries 8 and 9.

Lemma 4 leads to the definition of a multiplicity of new operations. We give one example which shows that these operations need not be related in any obvious way to Massey products $\left(\left\langle\alpha,{ }_{\beta_{2}, \gamma_{2}}^{\beta_{1} \gamma_{1}}\right\rangle\right.$ can be regarded as a "matric" Massey triple product).

EXAMPLE 12. Let $\alpha_{i} \in H(U), 1 \leqq i \leqq 7$. Suppose that $a_{i} \in U$ is a representative cycle for $\alpha_{i}$ and suppose there exist chains $b_{1}, b_{2}, c_{1}, c_{2}$ such that

$$
\begin{aligned}
& \delta b_{1}=\tilde{a}_{2} a_{3}+\tilde{a}_{4} a_{5}, \quad \delta b_{2}=\tilde{a}_{1} a_{2}+\tilde{a}_{6} a_{7}, \\
& \delta c_{1}=\bar{a}_{1} a_{4} \text { and } \delta c_{2}=\tilde{a}_{7} a_{3} .
\end{aligned}
$$


Observe that $\delta e=0$, where

$$
e=\bar{a}_{1} b_{1}+\bar{b}_{2} a_{3}+\bar{c}_{1} a_{5}+\bar{a}_{6} c_{2} .
$$

Under these hypotheses, we define $\varepsilon\left(\alpha_{1}, \cdots, \alpha_{7}\right)$ to be the set of all homology classes $\{e\}$ so obtainable. Then $\varepsilon\left(\alpha_{1}, \cdots, \alpha_{7}\right)$ is related to $\partial_{2} \theta$, where

$$
\left[\alpha_{1}\left|\alpha_{2}\right| \alpha_{3}\right]+\left[\alpha_{1}\left|\alpha_{4}\right| \alpha_{5}\right]+\left[\alpha_{6}\left|\alpha_{7}\right| \alpha_{3}\right] \in E^{1} U
$$

survives to 0 , by an analog of Theorem 6. Defining "good" operations $\varepsilon\left(\alpha_{1}, \cdots, \alpha_{7}\right)$ we can again obtain analogs of Corrollaries 8 and 9.

REMARKS 13. Stasheff [9] first noticed a similarity between Massey products and the differentials in $\left\{E^{r} U\right\}$. In an application to $H$-spaces, he called the differentials $\partial_{r}: E_{r+1, q}^{r} U \rightarrow E_{1, q+r-1}^{r} U$ "Yessam" operations, and showed that $\left\{E^{r} U\right\}$ could be defined under weaker assumptions than associativity on the product in $U$.

\section{REFERENCES}

1. J. F. Adams, On the structure and applications of the Stecurod algebra, Comment. Math. Helv. 32 (1958), 180-214.

2. M. G. Barratt, Homotopy operations and homotopy groups, Amer Math. Soc. Summer Topology Institute (Seattle), 1963.

3. H. H. Gershenson, Higher composition products, (to appear).

4. S. MacLane, Homology, Academic Press, New York, 1963.

5. W. S. Massey, Some higher order cohomology operations, Symposium International de Topologia Algebraica, Universidad Nacional Autónoma de México and UNESCO, 1958; 145-154.

6. J. P. May, The cohomology of restricted Lie algebras and of Hopf algebras, Bull. Amer. Math. Soc. 71 (1965), 372-377; J. of Algebra (to appear).

7. M. Mimura, On the generalized Hopf homomorphism and the higher composition. I, J. Math. Kyoto Univ. 4 (1964), 171-190.

8. K. Oguchi, A generalization of secondary composition and applications, J. Fac. Sci. Univ. Tokyo Sect. I 10 (1963-1964), 29-79.

9. J. D. Stasheff, Homotopy associativity of H-spaces. II, Trans. Amer. Math. Soc. 108 (1963), 293-312.

10. H. Toda, Generalized Whitehead products and the homotopy groups of spheres, J. Inst. Polyteck. Osaka City Univ. 3 (1952), 43-82.

11. C. T. C. Wall, Generators and relations for the Steenrod algebra, Ann. of Math. 72 (1960), 429-444.

YAIE UNIVERSITY,

New Haven, Connecticut 